\title{
Avaliação de modelo de difusão considerando a variação no tempo do teor de cloretos na superfície do concreto
}

\section{Evaluation of the diffusion model considering the variation in time of the chloride content of the concrete surface}

\author{
Cristiane Arpino Silva ${ }^{1}$, André Tavares da Cunha Guimarães ${ }^{2}$
}

\author{
${ }^{1}$ Universidade Federal do Rio Grande, Mestranda do curso de Pós-Graduação em Engenharia Oceânica. Av. \\ Itália, km 8, Campus Carreiros, 96201-900 - Rio Grande, RS, Brasil \\ e-mail: atco@vetorial net \\ ${ }^{2}$ Universidade Federal do Rio Grande, Escola de Engenharia, Laboratório de Engenharia Civil. Av. Itália, km \\ 8, Campus Carreiros, 96201-900 - Rio Grande, RS, Brasil \\ e-mail: crisarpino@ibest.com.br
}

\section{RESUMO}

Este trabalho objetiva avaliar o aumento da taxa de deposição de cloretos na superfície do concreto (Cs) exposto à ambiente marítimo, no tempo, e avaliar modelo de difusão que considera esta condição. Para tal, fora realizada análise e comparação entre os perfis de cloretos em tetrápodes localizados no extremo sul do Brasil, obtidos de amostras extraídas aos 5 anos (dezembro/2002) e 9,5 anos (junho/2007) de quatro micro climas, sendo estes localizados em diferentes condições de exposição no ambiente estudado. Na análise constatou-se que perfis com maiores idades, ou seja, a 9,5 anos apresentaram melhor definição nas curvas do que perfis a 5 anos. Foi utilizado um modelo para cada idade de perfil medido, considerando-se a variação de CS, pois se observou que este aumentou significativamente de 5 anos para 9,5 anos. A partir do modelo obtido com o perfil medido aos 5 anos estimou-se o perfil para a idade de 9,5 anos o qual foi comparado com perfil medido nesta idade. O mesmo procedimento foi realizado para o perfil medido aos 9,5 anos, ou seja, foi estimado o perfil para 5 anos e comparado com perfil medido na respectiva idade. Desta forma foi possível observar a eficiência do modelo utilizado, ou seja, que perfis de aproximadamente 5 anos ainda são de pouca idade para se usar como estimativa de vida útil residual de uma estrutura, mesmo considerando a variação de Cs. Já, perfis com aproximadamente 10 anos podem ser utilizados, sendo que estes ainda podem ter os valores de Cs aumentando no tempo. Além disso, o ambiente pesquisado demonstrou grande agressividade, atingindo percentuais de cloretos elevados. O que vem a ser importante, pois caso o concreto fosse armado, já teria atingido a despassivação do aço. Deve-se dar continuidade a esta pesquisa em idades maiores, avaliando o comportamento das curvas e a eficiência do modelo utilizado.

Palavras-chave: Durabilidade, Tetrápodes, Cloretos, Concreto, Difusão.

\section{ABSTRACT}

This work aims to evaluate the increase in the rate of chloride deposition on the concrete surface (Cs) exposed to marine environment, on time, and evaluate the diffusion model which considers this condition. For that, an analysis and comparison were undertaken among the chloride profiles in tetrapods located in the southern most area of Brazil, obtained from samples extracted at 5 years (December/2002) and 9,5 years (July/2007) from four micro environments, located in different exposure areas. The analysis showed that profiles at an older age, e.g. 9.5 years, present better definition in the curves than profiles at 5 years. A model has been developed for each age of measured profiles for each micro environment, taking into account the variation in Cs since it increased significantly from 5 to 9.5 years. The model obtained from the profile at 5 years 
was used to estimate the profile at the age of 9.5 years, which has been compared with the measured profile at this age. The same procedure was carried out to the profile measured at 9.5 years, that is, the profile at 5 years was estimated and compared with the profile measured at the same age. Thus it was possible to observe the efficiency of the utilised model: the profiles of approximately 5 years are inadequate to be used as an estimative of residual life cycle of a structure, even if the Cs variation is taken into account. On the other hand, profiles with approximately 10 years can be utilised considering that these profiles can still have their Cs values increased. Moreover, the environment investigated showed great aggressivity, reaching high chloride percentages. This is an important fact since, if the concrete is considered as reinforced, steel depassivation would have been reached. Future activities for this research include an older age, evaluating the behaviour of the curves and the efficiency of the suggested model.

Keywords: Durability, tetrapods, chlorides, concrete, diffusion.

\section{INTRODUÇÃO}

Algumas pesquisas $[\underline{1}, \underline{10}]$ demonstraram um aumento na taxa de deposição de cloretos na superfície do concreto (Cs) em função do tempo. Também, Meira [9] ao estudar essa taxa ao longo de meses, constatou que este comportamento condiciona-se, em sua maioria, aos tipos de atmosfera marinha nos quais encontra-se inserida a estrutura de concreto. Enfim, a importância deste estudo deve-se a grande influência de Cs na velocidade de penetração da frente de ataque dos íons cloreto, ou seja, quanto maior seu valor, maior será a velocidade de penetração desta frente de ataque, despassivando em menor tempo a barra de aço.

De acordo com Castro et al.[2] e Rincón et al.[12] a deposição desses íons desempenha um papel importante como um indicador ambiental na vida útil de estruturas de concreto em zona de atmosfera marinha. Além disso, através de algumas pesquisas é evidente que o valor de Cs tende a aumentar com a idade do concreto [14]. Neste sentido, se pode citar os estudos de Guimarães et al. [7] e Pereira [11], que ao estudarem estruturas offshore com mais de 20 anos constataram que Cs tende a aumentar com a idade do concreto, mas tende a estabilizar-se quando atinge um determinado valor máximo.

Crank [ㅁ] apresentou modelo considerando a variação de Cs, sendo avaliado, neste estudo, modelo em que o valor de Cs varia em função da raiz do tempo, para o ambiente pesquisado.

Nesta pesquisa foi estudada a dinâmica de penetração de íons cloreto no concreto ao longo do tempo em tetrápodes localizados na barra leste dos molhes de acesso ao complexo portuário da cidade do Rio Grande-RS-Brasil. Com essa finalidade foi realizado um monitoramento do comportamento de perfis de cloretos em dezembro/2002 (idade de 5 anos) e junho/2007.

Estes perfis foram tratados e utilizados modelos de penetração de cloretos considerando a variação de Cs. Após, os resultados obtidos através do modelo foram comparados com os perfis medidos in loco dos tetrápodes nas idades pesquisadas, onde foi avaliada a eficiência do modelo utilizado.

\section{MATERIAIS E MÉTODO}

\subsection{Modelos de penetração de cloretos considerando cs constante ou variável}

As equações 1 e 2 apresentadas por Crank [ㅁ] são dadas como solução da segunda lei de Fick considerando o coeficiente de difusão (D) e o teor de cloretos na superfície do concreto (Cs) constantes.

$$
C(x, t)=C_{0}+\left(C_{S}-C_{0}\right) \cdot \operatorname{erfc}\left(\frac{x}{(\sqrt{4 \cdot D \cdot t})}\right)
$$

onde:

$\mathrm{C}(\mathrm{x}, \mathrm{t})$ - concentração de íons cloreto em relação a massa de cimento na profundidade $\mathrm{x}$ a partir da superfície do concreto em dado tempo t $(\%)$;

$\mathrm{C} 0$ - concentração inicial de íons cloreto no interior do concreto $(\%)$;

CS - concentração superficial íons de cloreto, admitida constante (\%);

$\mathrm{x}$ - profundidade de penetração de íons cloreto $(\mathrm{mm})$;

$\mathrm{D}$ - coeficiente de difusão de íons cloreto ( $\mathrm{mm} 2 / \mathrm{ano}$ ), considerado constante;

$\mathrm{t}$ - tempo (anos); 
erfc - complementar da função erro de Gauss.

$$
M_{t}=2 \operatorname{Cs}\left(\frac{D_{\text {Csconst }} t}{\pi}\right)^{1 / 2}
$$

onde:

$\mathrm{M}_{\mathrm{t}}$ = massa total de cloretos que sofre difusão no tempo $\mathrm{t}(\%$.mm);

$\mathrm{D}_{\mathrm{Csconst}}=$ coeficiente de difusão de cloretos no concreto para Cs constante $\left(\mathrm{mm}^{2} / \mathrm{ano}\right)$;

Considerando a variação de Cs em função da raiz do tempo (equação 3) são apresentadas as equações 4 e 5 dadas por Crank [ $\underline{8}]$.

$$
\mathrm{C}_{\mathrm{x}=0}=\mathrm{Cs}=\mathrm{kt}^{1 / 2}
$$

onde:

Cs = concentração de íons cloreto na superfície do concreto - variável em função do tempo (\%);

$\mathrm{k}=$ parâmetro que considera a variação de Cs $\left(\%\right.$. ano $\left.{ }^{-1 / 2}\right)$;

$\mathrm{t}=$ tempo de exposição do concreto aos cloretos (anos).

$$
\mathrm{Mt}=\frac{1}{2} \mathrm{kt}\left(\pi \mathrm{D}_{\mathrm{Csvar}}\right)^{1 / 2}
$$

onde:

$\mathrm{D}_{\text {Csvar }}=$ coeficiente de difusão considerando Cs variável no tempo ( $\left.\mathrm{mm}^{2} / \mathrm{ano}\right)$.

$$
\begin{aligned}
& \mathrm{C}_{\mathrm{x}}=\mathrm{kt}^{1 / 2}\left\{\exp \left(-\frac{\mathrm{x}^{2}}{4 \mathrm{D}_{\text {Csvar }} \mathrm{t}}\right)-\frac{\mathrm{x} \pi^{1 / 2}}{2 \sqrt{\mathrm{D}_{\text {Csvar }} \mathrm{t}}} \operatorname{erfc} \frac{\mathrm{x}}{2 \sqrt{\mathrm{D}_{\text {Csvar }} \mathrm{t}}}\right\} \\
& =\mathrm{k}(\pi \mathrm{t})^{1 / 2} \operatorname{ierf} \frac{\mathrm{x}}{2 \sqrt{\mathrm{D}_{\text {Csvart }}}}
\end{aligned}
$$

Igualando os valores de Mt das equações 2 e 4 obtém-se que para um mesmo perfil de cloreto o coeficiente de difusão é $62 \%$ maior quando considera-se Cs constante do que para Cs variável em função de raiz do tempo. Portanto há uma diferença significativa entre os dois modelos.

\subsection{Estudo de caso}

Foram pesquisados quatro micro ambientes do molhe leste (Figura 1), situados na entrada do Canal do Rio Grande, no complexo portuário da cidade do Rio Grande-RS-Brasil. Cada micro ambiente estudado foi representado por dois tetrápodes, totalizando oito, cujas denominações foram as seguintes:

- MLLS - micro ambiente do molhe leste, voltado para leste e em cota superior;

- MLLM - micro ambiente do molhe leste, voltado para leste e em cota média;

- MLOM - micro ambiente do molhe leste, voltado para oeste e em cota média;

- MLOI - micro ambiente do molhe leste, voltado para oeste e em cota média no interior do molhe.

$$
\begin{gathered}
\text { Oeste } \\
\text { Leste Canal do Rio Grande }
\end{gathered}
$$

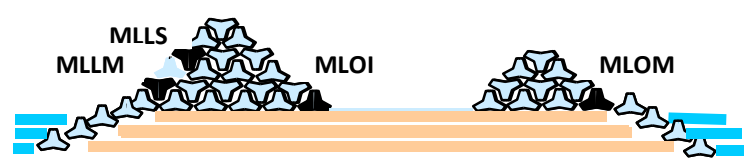

Figura 1: Localização dos micro ambientes no molhe leste da barra do Rio Grande

Os materiais utilizados na elaboração do concreto foram o agregado graúdo constituído de pedra granítica e o agregado miúdo de areia quartzosa. Foi utilizado cimento de alta resistência inicial e resistente a 
sulfatos contendo aproximadamente $12 \%$ de cinza volante, com resistência à compressão aos 28 dias de 48MPa. A relação a/c média foi de 0,5 , sendo que a resistência à compressão obtida aos 28 dias $\left(\mathrm{f}_{\mathrm{ck}}\right)$ foi de aproximadamente $32 \mathrm{MPa}[\underline{5}]$.

A água utilizada no concreto apresentou as seguintes características: teores de cloretos de aproximadamente $430 \mathrm{mg} / \mathrm{l}$, teores de sulfatos de $100 \mathrm{mg} / \mathrm{l}, \mathrm{pH}$ de 7,3, resíduo sólido de $250 \mathrm{mg} / \mathrm{l}$ e matéria orgânica em $\mathrm{O} 2$ consumido de 1,2 mg/l. Conforme os relatórios de obra existentes não foi previsto o uso de aditivos.

As amostras foram obtidas com furadeira especial e o material foi extraído em camadas de $5 \mathrm{~mm}$ desde a superfície externa até a profundidade de $50 \mathrm{~mm}$, sendo que os materiais da mesma camada de dois tetrápodes constituíram uma amostra, cujo resultado representou o teor médio de cloreto para cada micro ambiente pesquisado. A partir dos teores destas amostras, obtidos nas idades de 5 anos e 9,5 anos, foram traçados os perfis de cloretos.

\subsection{Método de aplicação do modelo}

Após o traçado dos perfis de medição de cloretos, através da equação 1, obteve-se a regressão dos perfis de cloretos, sendo esta equação referente à segunda lei de Fick, considerando Cs constante. É importante salientar que esta equação foi utilizada somente com a intenção de se obter uma melhor definição dos perfis de medição, assim como estimar o valor de Cs nas idades estudadas.

A partir dos resultados observou-se uma grande variação de CS ao longo do tempo, o qual ajustou-se, aproximadamente, em função da raiz quadrada do tempo, sendo que a partir da equação 3 foi estimado o parâmetro k. Calculando a área abaixo de cada perfil de medição de cloreto obteve-se os valores de Mt, e utilizando a equação 4 calculou-se $\mathrm{D}_{\mathrm{CS} v a r}$ para cada idade pesquisada.

Na tentativa de obter perfis em diversas idades, através da equação 5 foram estimados novos perfis de cloretos. No entanto, estes perfis apresentaram comportamento não satisfatório, pois os percentuais de cloretos reduziram da superfície até uma determinada profundidade, aumentando para profundidades maiores. Assim, para representar os perfis de cloretos em diferentes idades utilizou-se os mesmos valores de Mt e Cs obtidos inicialmente, entretanto a curva obtida foi ajustada a uma função erro de Gauss. A partir desse valor e do uso da equação 1 pôde-se representar o perfil para cada idade pesquisada.

Tendo como referência os trabalhos de Guimarães et al. [7] e Pereira [11], optou-se por considerar para os tetrápodes um valor máximo de Cs de 3,5\% em relação a massa de cimento. Após, foi elaborada uma estimativa dos perfis de cloretos para idades em que se supõe que o concreto já tenha atingido esse valor máximo e a partir destas curvas, foram estimados perfis para idades maiores. É importante salientar que o tempo em que se atinge o referido Cs máximo foi denominado tempo de troca $\left(\mathrm{t}_{\text {troca }}\right)$, ou seja, quando ocorre a troca o modelo considerando CSvariável ( $\mathrm{C}_{\text {Svar }}$ ) para CS constante $\left(\mathrm{C}_{\text {Sconst }}\right)$ (Figura 2). Na obtenção de cada perfil, em cada tempo de troca foi calculado um tempo equivalente $\left(\mathrm{t}_{\mathrm{eq}}\right)$, que é o tempo necessário para atingir o perfil no tempo de troca considerando Cs constante. Portanto, a partir da igualdade entre as equações 2 e 4 , obteve-se a equação 6 (Figura 2).

$$
t_{\text {troca }}=1,62 . t_{\text {eq }}
$$

onde:

$\mathrm{t}_{\text {troca }}=$ tempo para o qual $\mathrm{C}_{\mathrm{S}}$ é máximo, a partir do qual se considera $\mathrm{C}_{\mathrm{S}}$ constante e igual a 3,50\% em relação a massa de cimento;

$\mathrm{t}_{\mathrm{eq}}=$ tempo para o qual se ajusta o perfil considerando $\mathrm{C}_{\mathrm{S}}$ variável e o perfil considerando $\mathrm{C}_{\mathrm{S}}$ constante. 


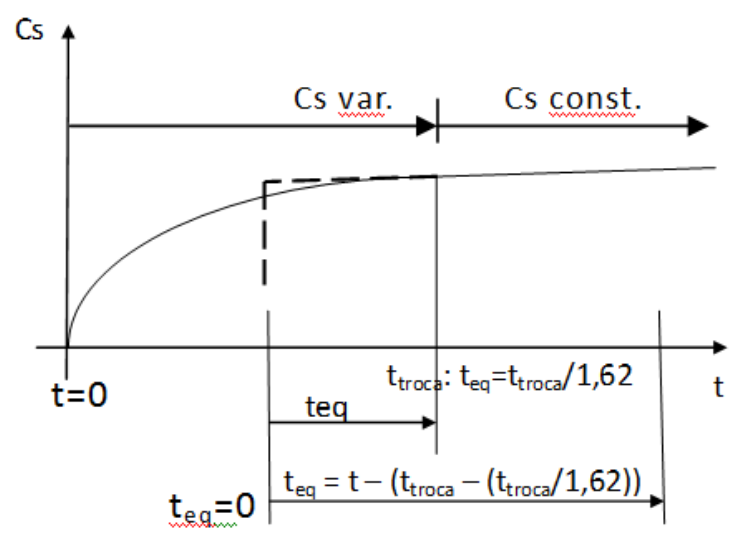

Figura 2: Comportamento de Cs ao longo do tempo

Para idades superiores a $t_{\text {troca }}$ foi calculado tempo equivalente $\left(t_{\text {eq }}\right.$ '), conforme equação 7 e Figura 2.

$$
\text { teq }^{\prime}=\mathrm{t}-\left(\mathrm{t}_{\text {troca }}-\left(\frac{\mathrm{t}_{\text {troca }}}{1,62}\right)\right.
$$

sendo:

$\mathrm{t}_{\mathrm{eq}}$ ' $=$ tempo equivalente para $\mathrm{t}>\mathrm{t}_{\text {troca }}$

Todos os procedimentos descritos acima foram realizados tanto para os perfis medidos aos 5 anos, como para os perfis medidos aos 9,5 anos, tendo sido considerado um k para cada idade pesquisada. Entretanto também, por regressão, estimou-se um keq, o qual apresentou o menor erro quadrado na estimativa de CS variável entre as idades estudadas. Portanto, com keq foram repetidos os mesmos procedimentos utilizados com k de 5 anos e 9,5 anos.

Com o modelo obtido para perfis de 5 anos foram estimados os perfis aos 9,5 anos, os quais foram comparados com os perfis de cloretos medidos aos 9,5 anos. $\mathrm{O}$ mesmo procedimento foi realizado com o modelo obtido para perfis de 9,5 anos, ou seja, a partir destas curvas foram estimados perfis aos 5 anos que foram comparados com perfis medidos nesta idade. Desta forma pôde-se verificar a eficiência do modelo utilizado.

A fim de comparar o desempenho de cada modelo obtido definiu-se um erro relativo como sendo:

$$
\mathrm{E}_{\text {Relativo }}=\frac{\mathrm{E}_{\text {Est. }}}{\mathrm{E}_{\text {Min Reg. }}}
$$

onde:

$\mathrm{E}_{\mathrm{Est} .}=$ Erro quadrado da estimativa (entre pontos medidos e pontos estimados pelo modelo);

$\mathrm{E}_{\text {Mín.Reg. }}=$ Erro quadrado mínimo da regressão (entre pontos medidos e pontos de sua regressão);

$\mathrm{E}_{\text {Relativo }}=$ Erro relativo .

Portanto, para que o modelo proposto tivesse um bom desempenho este deveria apresentar um pequeno erro relativo.

\section{RESULTADOS E DISCUSSÕES}

Nas Figuras 3 e 4 são apresentados os perfis de cloretos para os micro ambientes MLLS; MLOI e MLOM; MLLM, respectivamente, considerado k de 5 anos e de 9,5 anos. Nas Figuras 5 e 6 são apresentados os perfis dos mesmos pontos, porém para keq, respectivamente. 
ARPINO, C.R.; GUIMARÃES, A.C.T. revista Matéria, v.19, n.02, pp. 81-93, 2014.

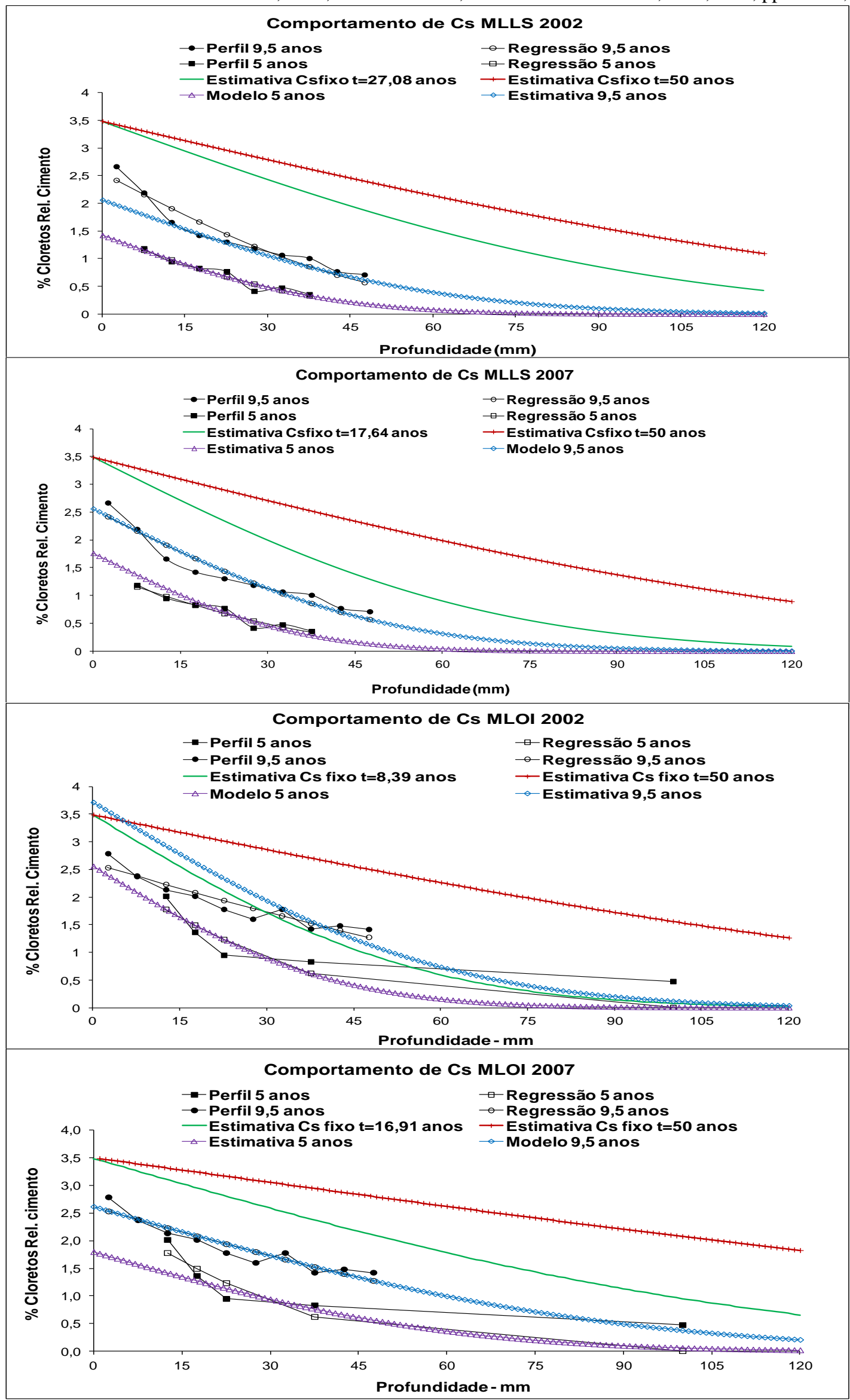

Figura 3: Perfis de Cl- para modelo de 5 anos (k5anos) e modelo de 9,5 anos (k9,5anos), para MLLS e MLOI. 
ARPINO, C.R.; GUIMARÃES, A.C.T. revista Matéria, v.19, n.02, pp. 81-93, 2014.

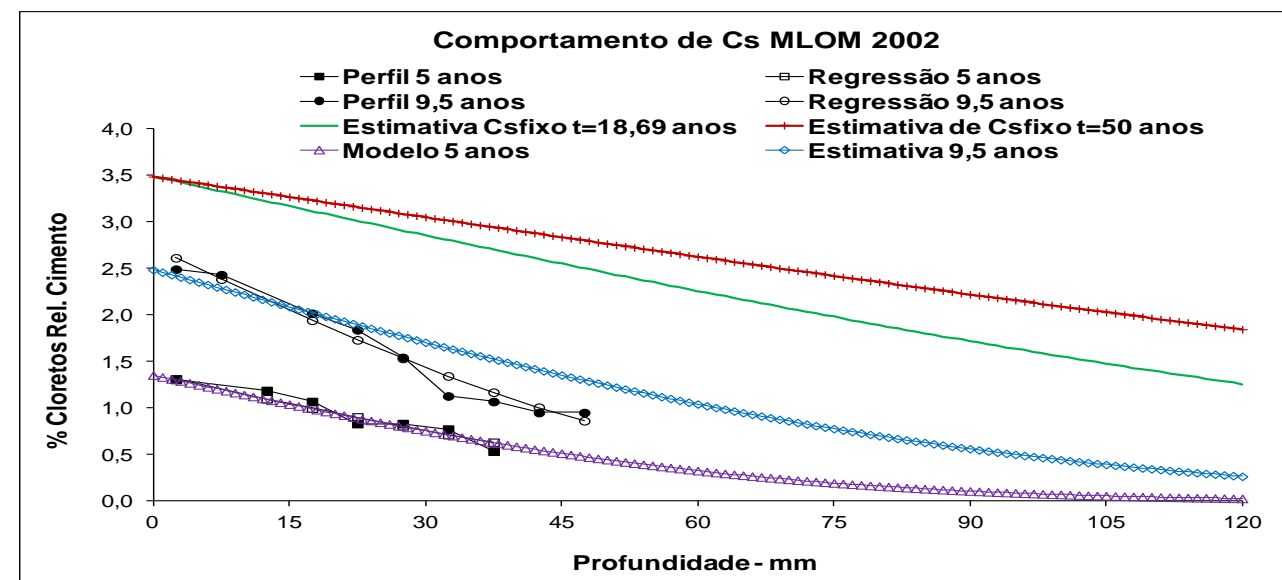

Comportamento de Cs MLOM 2007

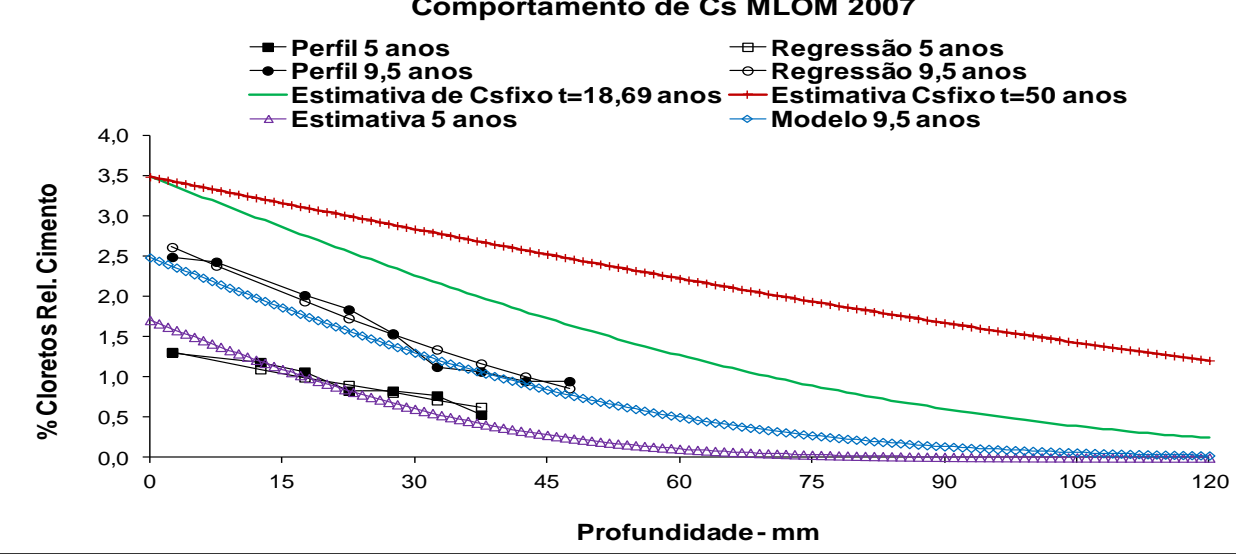

Comportamento de Cs MLLM 2002
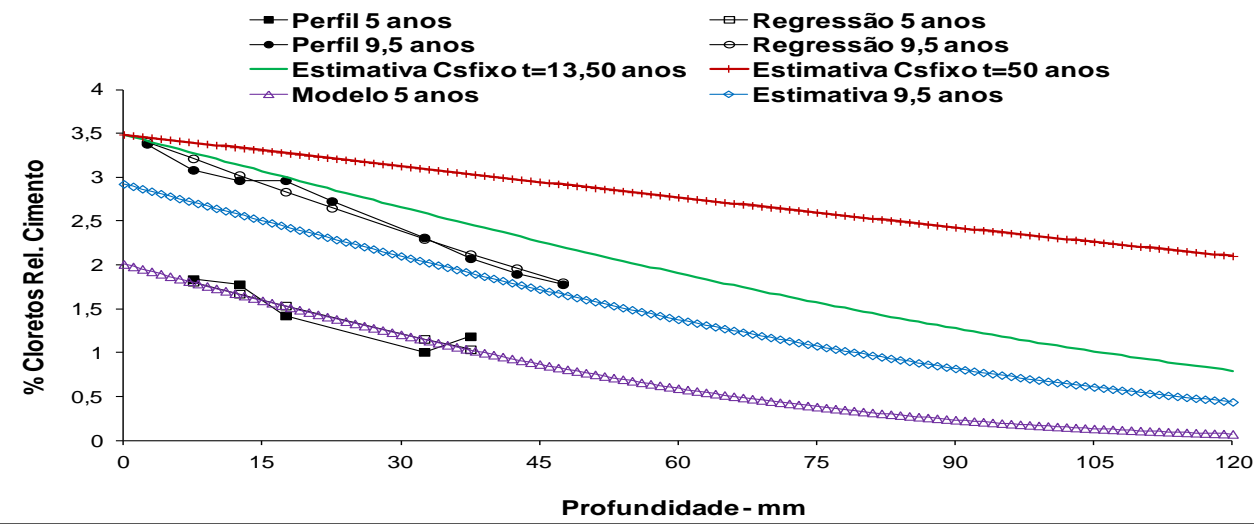

Comportamento de Cs MLLM 2007

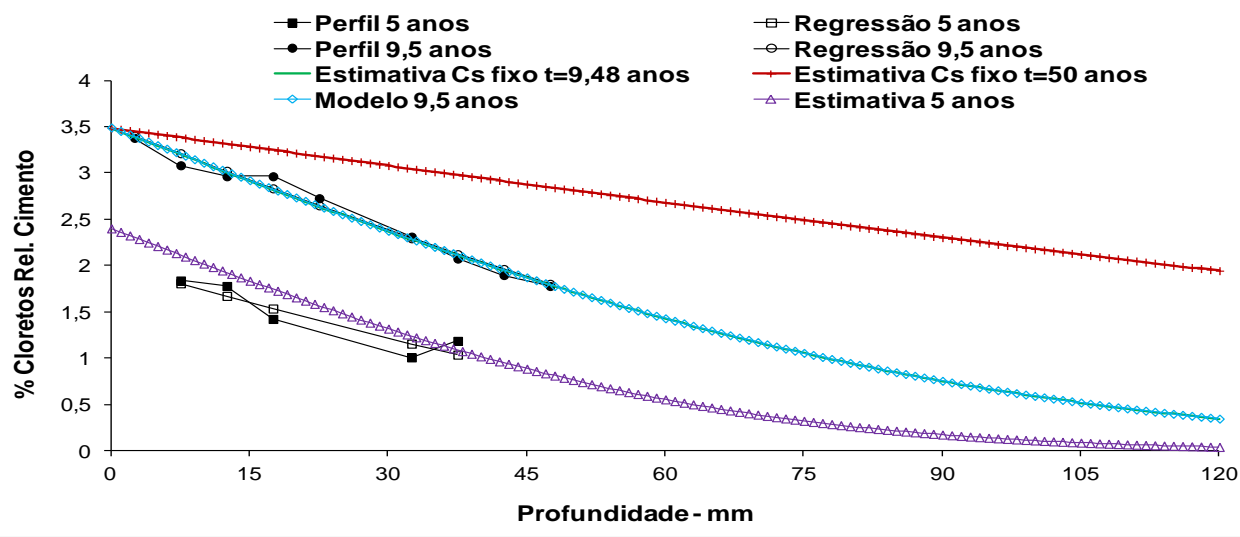

Figura 4: Perfis de Cl- para modelo de 5 anos (k5anos) e modelo de 9,5 anos (k9,5anos), para MLOM e MLLM. 
ARPINO, C.R.; GUIMARÃES, A.C.T. revista Matéria, v.19, n.02, pp. 81-93, 2014.
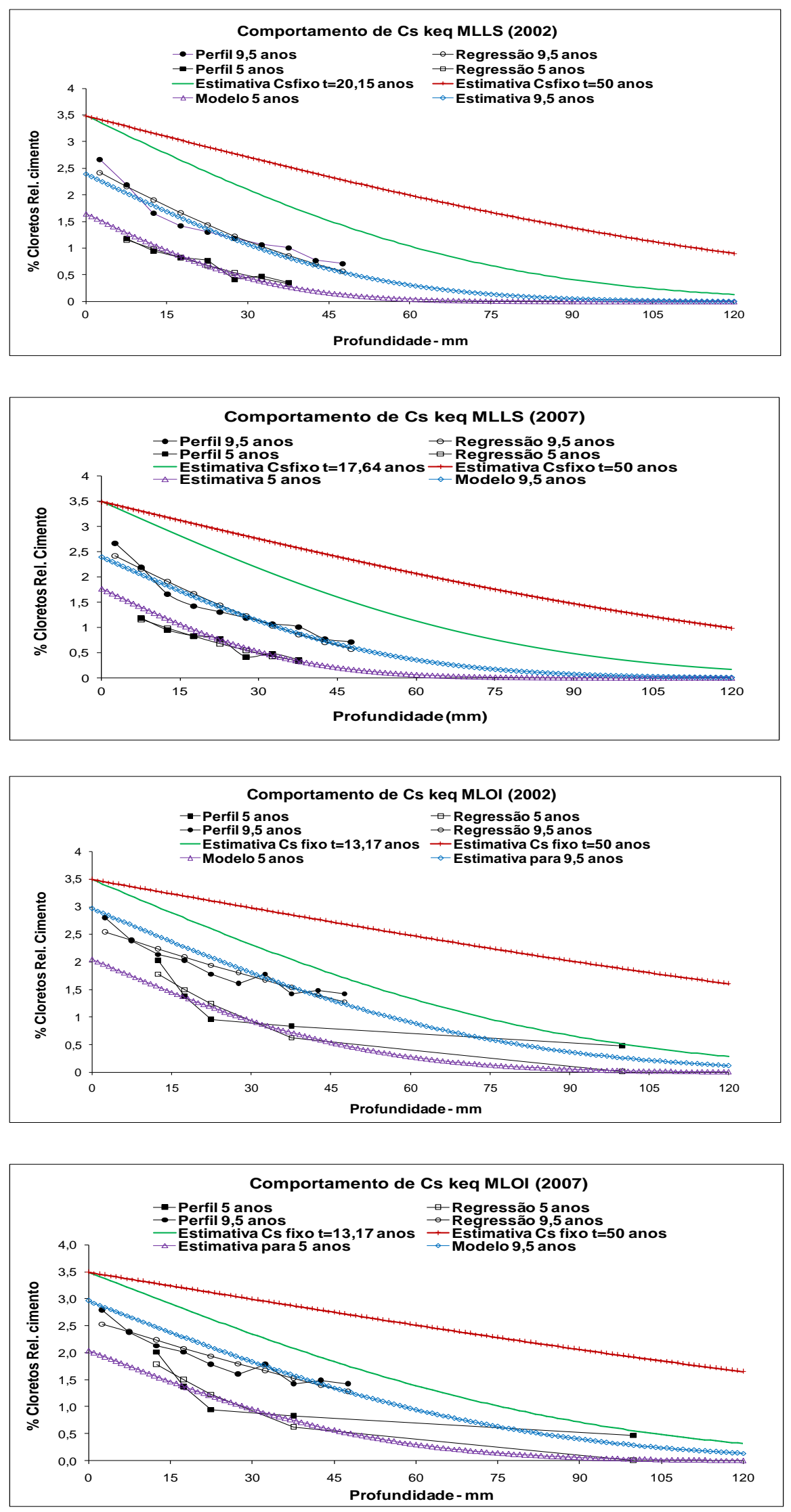

Figura 5: Perfis de Cl- para modelo de 5 anos (keq) e modelo de 9,5 anos (keq), para MLLS e MLOI. 
ARPINO, C.R.; GUIMARÃES, A.C.T. revista Matéria, v.19, n.02, pp. 81-93, 2014.

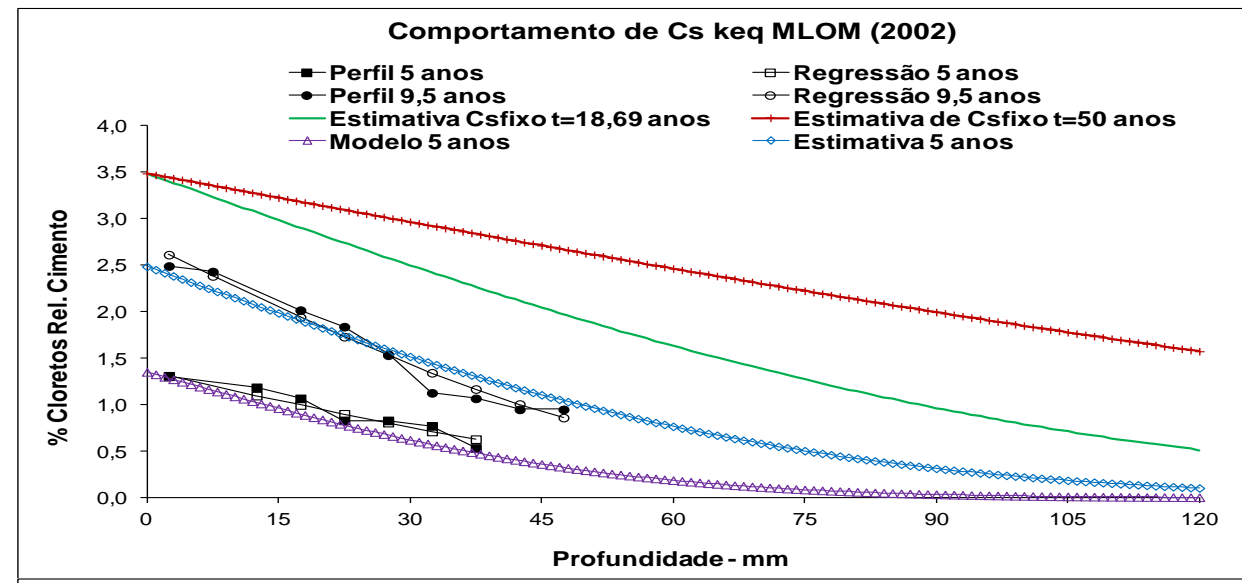

Comportamento de Cs keq MLOM (2007)

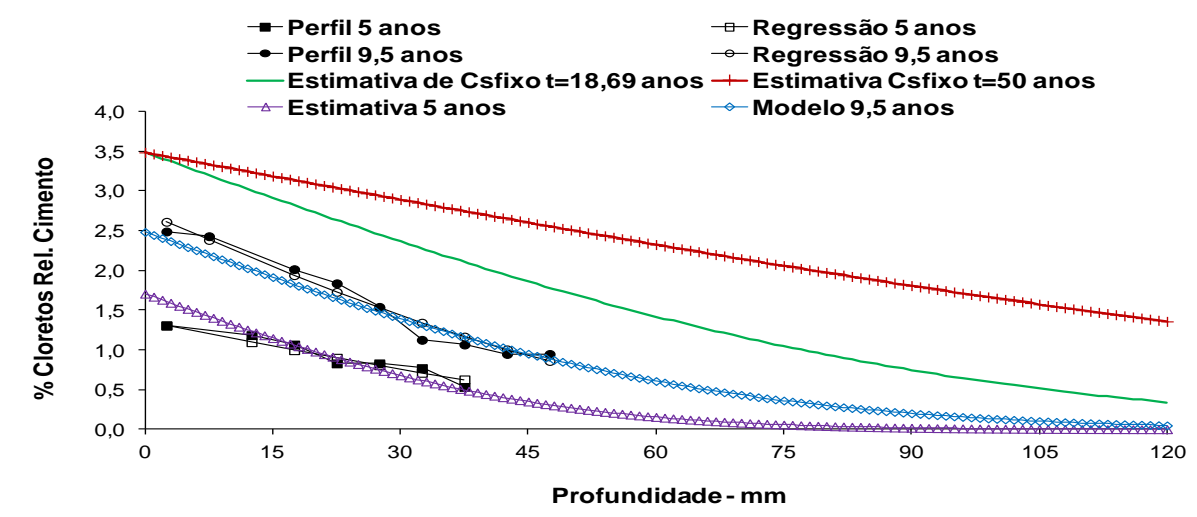

Comportamento de Cs keq MLLM (2002)
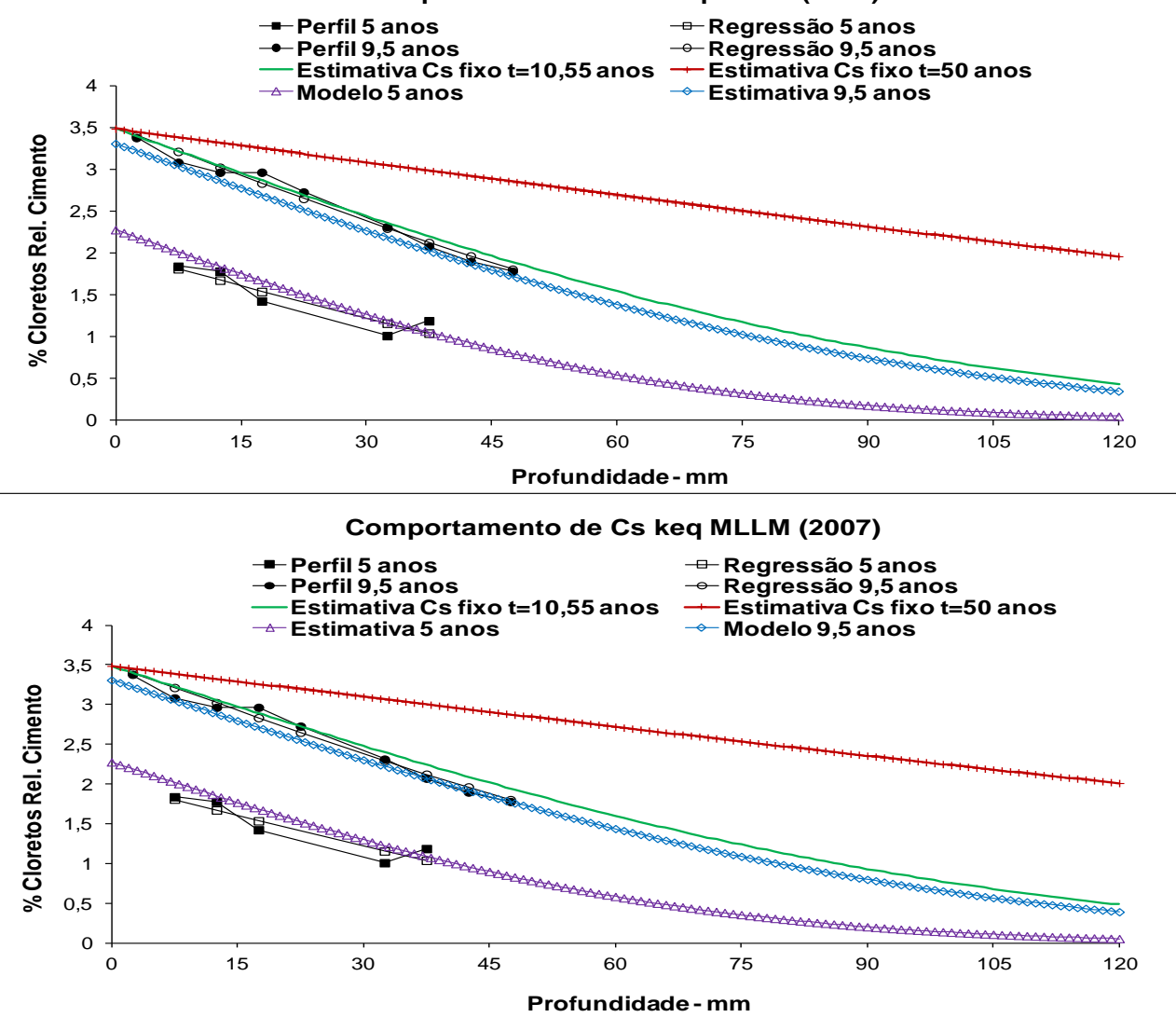

Figura 6: Perfis de Cl- para modelo de 5 anos (keq) e modelo de 9,5 anos (keq), para MLOM e MLLM. 
ARPINO, C.R.; GUIMARÃES, A.C.T. revista Matéria, v.19, n.02, pp. 81-93, 2014.

Na Tabela 1 são apresentados os parâmetros estimados para os 4 micro-ambientes pesquisados.

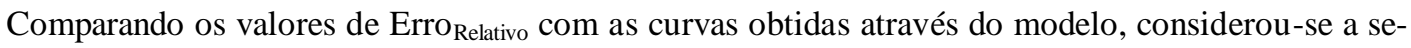
guinte classificação para melhor analisar os resultados obtidos: entre 1 e 2 - muito bom (MB); entre 2 e 4 bom (B); entre 4 e 10 - regular (Reg); e maior que 10 - ruim(R).

Tabela 1: Parâmetros estimados para os quatro microambientes pesquisados dos tetrápodes

\begin{tabular}{|c|c|c|c|c|c|c|}
\hline Microamb. & $\mathrm{Cs}_{5 \text { anos }} \%$ & $\mathrm{Cs}_{9,5 \text { anos }} \%$ & $\begin{array}{c}\mathrm{D}_{5 \text { anos }-\mathrm{t}_{\text {troca }}} \\
\mathrm{E}_{\text {relativo }} \mathrm{mm}^{2} / \mathrm{ano}- \\
\text { ano }\end{array}$ & $\begin{array}{c}\mathrm{D}_{9,5 \text { anos }-\mathrm{t}_{\text {troca }}} \\
\mathrm{E}_{\text {relativo }} \mathrm{mm}^{2} / \mathrm{ano}- \\
\text { ano }\end{array}$ & $\begin{array}{c}* \mathrm{D}_{5 \text { anos }-} \mathrm{t}_{\text {troc }} \mathrm{E}_{-} \\
\text {relativo } \\
\mathrm{mm}^{2} / \text { ano }- \text { ano }\end{array}$ & $\begin{array}{c}* \mathrm{D}_{9,5 \text { anos }-} \mathrm{t}_{\text {troca }} \mathrm{E}_{\text {re- }} \\
\text { lativo } \\
\mathrm{mm}^{2} / \text { ano }- \text { ano }\end{array}$ \\
\hline MLLS & 0,24 & 0,43 & $179 / 27-2,7$ & $129 / 18-3,4$ & $133 / 20-1,2$ & $147 / 20-2,2$ \\
\hline MLLM & 0,34 & 0,59 & $593 / 14-22,9$ & $452 / 9-3,9$ & $465 / 11-3,6$ & $503 / 11-2,4$ \\
\hline MLOM & 0,23 & 0,46 & $470 / 30-10,7$ & $189 / 16-9,1$ & $296 / 19-2,8$ & $227 / 19-4,4$ \\
\hline MLOI & 0,43 & 0,44 & $185 / 8-15,1$ & $399 / 17-1,4$ & $291 / 13-2,5$ & $311 / 13-1,1$ \\
\hline
\end{tabular}

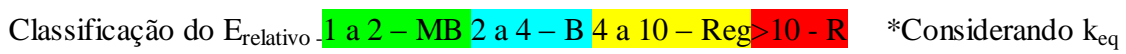

\subsection{Discussão dos resultados}

Analisando os perfis de medição aos 9,5 anos observou-se uma melhor definição das curvas, pois perfis de 5 anos ainda apresentaram picos ou oscilações que desapareceram na idade de 9,5 anos. O perfil que apresentou uma melhor definição aos 5 anos foi o micro-ambiente MLLS o qual não apresentou pico ou oscilação nessa idade e demonstrou comportamento nos perfis de cloretos de 5 anos e 9,5 anos muito próximos, sendo que o micro-ambiente MLLM apresentou um pequeno pico aos 5 anos. Já o micro-ambiente MLOM apresentou uma oscilação no perfil de 5 anos. O ponto MLOI apresentou um grande teor em uma profundidade considerável, ou seja, aos 100 mm, com um teor de cloretos de 0,47\% em relação à massa do cimento, valor esse, em que o aço já teria atingido a despassivação, caso o concreto dos tetrápodes fosse armado.

O teor de cloretos na superfície do concreto (Cs) aumentou de 5 para 9,5 anos, considerando os 4 micro ambientes pesquisados, assim como o coeficiente de difusão (D) que diminuiu de 5 para 9,5 anos. Isso já era esperado, pois Cs tende a aumentar com tempo até atingir um determinado valor máximo. Esse comportamento torna-se evidente ao observar a tabela 1, exceto para MLLM, que possivelmente já tenha atingido o máximo teor fixo de cloretos de 3,5\% em relação à massa do cimento, valor esse comum para obras com mais de 20 anos de exposição, conforme foi proposto por Guimarães et al. [7] e Pereira [11].

De maneira geral, o teor de cloretos na superfície do concreto - Cs - aumentou com o passar do tempo (Tabela 1). Por outro lado, os perfis de 9,5 anos demonstraram melhor definição, pois os perfis de 5 anos ainda apresentaram picos ou oscilações os quais desapareceram aos 9,5 anos. Talvez possa ser este o motivo de alguns micro climas apresentarem diferenças consideráveis entre os perfis estimados para 9,5 anos (quando se utilizou o modelo baseado nos perfis medidos aos 5 anos) e os perfis medidos aos 9,5 anos.

Nota-se que na idade em que o valor de Cs é maior, a profundidade de ataque de cloretos no concreto, também é maior, o que é coerente com os modelos de previsão de vida útil []].

Conforme os resultados obtidos nesta pesquisa, perfis de aproximadamente 5 anos ainda são de pouca idade para se usar como estimativa de vida útil residual de uma estrutura de concreto, mesmo considerando a variação de Cs. Já, perfis com aproximadamente 10 anos podem ser utilizados, mas deve-se prever que estes ainda podem ter seus valores de Cs aumentando com o tempo. De modo geral, obteve-se um melhor comportamento dos perfis de estimativa quando se utilizou um keq do que no uso do $\mathrm{k}$ da respectiva idade. Isso foi confirmado através dos resultados obtidos nas figuras 3 e 4, onde as estimativas a 9,5 anos ficaram um pouco distantes dos perfis de medição in loco nessa idade. O mesmo confirmou-se através dos valores de Erelativo (Tabela 1) onde as piores estimativas foram quando se considerou o perfil de 5 anos, exceto para MLLS que apresentou estimativa a 9,5 anos boa.

Observa-se que perfis de estimativa aos 5 anos, obtidos a partir de perfis medidos a 9,5 anos tanto para $\mathrm{k}$ da idade como para keq apresentaram, de modo geral, maior similaridade, seja pelo comportamento ou inclinação das curvas (Figuras 3, 4, 5 e 6); ou mesmo pelos resultados apresentados na tabela 1, onde os 4 micro ambientes estudados apresentaram Erelativo de B a MB, exceto para MLOM que apresentou Erelativo regular. 
$\mathrm{Na}$ análise dos perfis de cloretos ficou evidente que perfis aos 9,5 anos apresentaram uma melhor definição das curvas do que perfis a 5 anos.

Além disso, foi verificada não só a importância do modelo utilizado como também a agressividade do ambiente pesquisado. É importante salientar que conforme os resultados obtidos nessa pesquisa e nos trabalhos de Guimarães [ㅁ] e Rodrigues [13] o cimento ARI-RS, somente com adição de $12 \%$ de cinza volante, não é recomendável para obras marítimas. Como exemplo pode-se citar o micro ambiente MLOI que apresentou um teor de cloretos elevado em uma profundidade considerável em apenas 5 anos de exposição do concreto, o que provavelmente já teria causado a despassivação precoce do aço, caso fosse utilizado concreto armado ao invés de concreto massa.

Costa e Appleton [3] realizaram estudos com concreto Portland Comum, onde, através de modelo baseado na segunda lei de Fick, obtiveram uma grande variação do coeficiente de difusão (D), chegando a variação de 3,4 vezes entre 6 meses e 36 meses, para zona de maré, sendo que entre 12 meses e 36 meses a variação foi de 1,47 vezes e de 24 meses para 36 meses de 1,23. Estes perfis apresentavam grande variação de Cs com o tempo. Com a intenção de manter estes valores, foram considerados valores de D conforme as equações 9,10 e 11.

$$
\begin{aligned}
& \mathrm{D}(\mathrm{t})=\mathrm{D}_{1} \cdot \mathrm{t}^{\mathrm{t}} \\
& \mathrm{C}_{\mathrm{S}}(\mathrm{t})=\mathrm{C}_{\mathrm{t}} \cdot \mathrm{t}^{\mathrm{n}} \\
& C(x, t)=C_{1} \cdot t^{n} \cdot\left(1-\operatorname{erf}\left\{x \mid 2 \cdot \sqrt{D_{1} \cdot t^{1-m}}\right\}\right)
\end{aligned}
$$

Onde $\mathrm{D}_{1}$ e $\mathrm{C}_{1}$ são respectivamente os valores de coeficiente de difusão e teor de cloreto na superfície do concreto após um ano. Considerando a variação sazonal, ou seja, as medições a cada ano, não era de se esperar grandes variações do coeficiente de difusão do concreto após um ou dois anos em concreto executado com cimento Portland comum, o que não ocorreu com o modelo proposto por Costa e Appleton [4].

As equações 3, 4 e 5 apresentadas por Crank [8] , obtidas através da segunda lei de Fick, considerando Cs variando no tempo e D constante, apresentaram boa estimativa, conforme o presente trabalho. Para estas equações quando se considera um perfil de uma determinada idade, ao projetar este perfil para idades futuras, mantém-se o valor de D. Quando tratados perfis de diferentes idades, no presente trabalho, observouse diferenças de D, diferenças essas menores que $20 \%$ em relação ao valor médio para os pontos MLLM e MLLS. Convém salientar que estas diferenças podem estar relacionadas às variações nos ensaios quando na obtenção dos perfis de cloretos. Já para os pontos MLOI e MLOM estas diferenças foram bem maiores, sendo que para o ponto MLOI o perfil obtido aos 5 anos foi irregular, apresentando inclusive um valor de D menor aos 5 anos em relação aos 9,5 anos. Já o ponto MLOM não se tem justificativa para tal diferença, mas as estimativas feitas de uma idade para outra, considerando D constante, conforme tabela 1, foram ruins de 5 anos para 9,5 anos, mas regular (MLOM) ou boa (MLOI) para estimativas de 9,5 anos para 5 anos. Isto, possivelmente se deve ao fato de que na idade de 5 anos os perfis, com baixos teores de cloreto e com grande parte da massa destes cloretos localizando-se próximo a superfície, sofrem mais a influência de outros fatores, como secagem e molhagem. Outro possível problema é ter utilizado nesta pesquisa valor de "n" da equação 10 igual a 0,5, podendo ser necessário ajuste deste valor, considerando os diversos micro ambientes pesquisados, conforme demonstram Costa e Appleton [4]. Portanto, considerou-se mais lógico utilizar as equações de Crank [] ] ao invés do modelo proposto por Costa e Appleton [4]].

Entretanto, deve-se dar continuidade a essa pesquisa obtendo perfis de cloretos em idades maiores, ou seja, em 15 ou 20 anos de exposição dos tetrápodes, na tentativa de avaliar o comportamento das curvas e a eficiência do modelo utilizado neste trabalho. Assim como, verificar se os valores de Cs ainda aumentam com o tempo, ou já possam ter atingido o valor máximo sugerido.

Convém ressaltar que as equações de Crank [] $]$ aplicam-se principalmente à avaliação de estruturas de concretos de pequena idade, caso em que a variação de Cs apresenta significativa influência.

Salienta-se que os modelos obtidos nesta pesquisa, através das equações de Crank [ㅁ], aplicam-se a ambientes e materiais similares aos citados neste trabalho.

\section{CONCLUSÕES}

$\mathrm{Na}$ análise dos perfis de cloretos ficou evidente que perfis aos 9,5 anos apresentam melhor definição das curvas do que perfis a 5 anos. Portanto, perfis de aproximadamente 5 anos ainda são de pouca idade para se usar 
ARPINO, C.R.; GUIMARÃES, A.C.T. revista Matéria, v.19, n.02, pp. 81-93, 2014.

como estimativa de vida útil residual de uma estrutura, mesmo considerando a variação de Cs. Portanto, perfis com aproximadamente 10 anos podem ser utilizados, mas deve-se prever que estes ainda podem ter seus valores de Cs aumentando no tempo. Além disso, foi verificado não só a importância do modelo utilizado como também a agressividade do ambiente pesquisado.

A exemplo disso deve-se destacar o micro ambiente MLOI, que apresentou perfis com teores de cloretos elevados a uma profundidade considerável, e com apenas 5 anos de exposição, o que provavelmente já teria causado a despassivação precoce do aço, caso esse concreto fosse armado.

De modo geral, se obteve um melhor comportamento dos perfis de estimativa quando se utilizou um keq (coeficiente de variação de Cs) em relação ao uso do k da respectiva idade.

Convém salientar a importância da continuidade dessa pesquisa, ou seja, de obter perfis de cloretos para maiores idades, não só na estrutura pesquisada neste estudo como em outras estruturas, na tentativa de avaliar o comportamento das curvas e a eficiência do modelo utilizado, assim como os valores de Cs, se ainda crescem com a idade ou já possam ter atingido o valor máximo sugerido.

\section{AGRADECIMENTOS}

À CAPES-Fundação Coordenação de Aperfeiçoamento de Pessoal de Nível Superior - pela concessão de apoio financeiro durante a realização desse trabalho.

\section{BIBLIOGRAFIA}

[1]CASTRO, P.,"Propuesta de un Modelo de Difusión de Cloruros en las Estructuras de Concreto Armado Expuestas en la Península de Yucatán”, In: Anais do $2^{\text {nd }}$ NACE - Latin American Region Corrosion Congress, México, 1996.

[2] CASTRO, P., RINCÓN, O.T., PAZINI, E.J., "Interpretation of chloride profiles from concrete exposed to tropical marine environments”, Cement and Concrete Research, v. 31, Issue 4, pp. 529-537, 2001.

[3] COSTA, A, APPLETON, J., "Chloride penetration into concrete in marine environment - Part I: Main parameters affecting chloride penetration”, Materials and Structures, v. 32, pp. 252-259. May. 1999.

[4] COSTA, A, APPLETON, J., "Chloride penetration into concrete in marine environment - Part II: Prediction of long term chloride penetration”, Materials and Structures, v. 32, pp. 354-359 June. 1999.

[5] FURG-FUNDAÇÃO UNIVERSIDADE DO RIO GRANDE, CENTRO DE PESQUISAS E ORIENTAÇÃO INDUSTRIAL- Relatórios de ensaios realizados durante a execução dos tetrápodes. Rio Grande, Rio Grande do Sul, 1997-1998.

[6] GUIMARÃES, A.T.C. Vida útil de estruturas de concreto armado em ambientes marítimos, Tese de D.Sc., Escola Politécnica da Universidade de São Paulo, Departamento de Engenharia de Construção Civil, PCC/USP - Universidade de São Paulo, São Paulo, Brasil, 2000.

[7] GUIMARÃES, A.T.C., CASTRO, P., NUNES, J.L.O., "Teor de Cloretos Próximos a Superfície do Concreto em Ambientes Marítimos", In: Anais do $49^{\circ}$ Congresso Brasileiro do Concreto, Bento Gonçalves, Rio Grande do Sul, Brasil, 2007.

[8] J. CRANK. The Mathematics of Diffusion, 2 ed., Oxford, UK, Oxford Univ. Press, 1975.

[9] MEIRA, G.R., Agressividade por Cloretos em Zona de Atmosfera Marinha Frente ao Problema da Corrosão em Estruturas de Concreto Armado, Tese de D.Sc., Programa de Pós-Graduação em Engenharia Civil, Universidade Federal de Santa Catarina, Florianópolis, Santa Catarina, Brasil, 2004.

[10] MEIRA, G.R., ANDRADE, C., PADARATZ, I.J., et al., "Chloride penetration into concrete structures in the marine atmosphere zone - Relationship between deposition of chlorides on the wet candle and chloride accumulated into concrete”, Cement and Concrete Composites, v. 29, Issue 9, pp. 667-676, 2007.

[11] PEREIRA, A.C., Métodos Probabilísticos para la Predicción de la Vida Útil de Estructuras de Hormigón: Influencia del Factor Variabilidad Espacial en el Caso de Plataformas Offshore en Brasil, Tesis de D.Sc., Universidad Politécnica de Madrid, Escuela Técnica Superior de Ingenieros de Caminos, Canales Y Puertos, Madrid, España, 2003. 
ARPINO, C.R.; GUIMARÃES, A.C.T. revista Matéria, v.19, n.02, pp. 81-93, 2014.

[12] RINCÓN, O.T. de., CASTRO, P., MORENO, E.I., et al, "Chloride profiles in two marine structures meaning and some predictions", Building and environment, v. 39, Issue 9, pp. 1065-1070, 2004.

[13] RODRIGUES, F.T., Medição do Grau de Saturação e a Difusão de Íons Cloreto em Concretos Executados com Cimento de Alta Resistência Inicial, Tese de M.Sc., Universidade Federal do Rio Grande, Rio Grande, Rio Grande do Sul, RGS, Brasil, 2009.

[14] SILVA, C.A., Comportamento dos perfis de cloretos em tetrápodes localizados nos molhes da barra (Rio Grande - RS - Brasil), Tese de M.Sc., Universidade Federal do Rio Grande, Rio Grande, Rio Grande do Sul, RGS, Brasil, 2010. 